\title{
IMPLANTAÇÃO DO SISTEMA DE CLASSIFICAÇÃO DE RISCO SUL-AFRICANO NO SERVIÇO DE URGÉNCIA E EMERGÉNCIA DE UM HOSPITAL QUARTENÁRIO E FILANTRÓPICO DA REGIÃO DE CURITIBA
}

\author{
Implementation of the South African Risk \\ Classification System in the urgency and emergency \\ service of a quaternary and philanthropic hospital in \\ the region of the city of Curitiba
}

Priscilla de Almeida Cunico', Eliane Cristina Sanches Maziero²

1. Enfermeira. Especialista em Segurança do Paciente para Profissionais/FioCruz. Setor de Gestão de Processos e Segurança do Paciente, Hospital Nossa Senhora das Graças. ORCID:

2. Enfermeira. Doutora. Escola de Saúde Pública do Paraná. ORCID: https://orcid.org/0000-00031264-0833

CONTATO: Priscilla de Almeida Cunico | Rua Presidente Rodrigo Otavio, 777 | Alto da Rua XV | CEP 80045395 | Curitiba | Paraná | Telefone: (41) 99218-1314 | E-mail: priscilla_cunico@ yahoo.com.br

COMO CITAR Cunico PL, Maziero ECS. Implantação do sistema de classificação de risco sul-africano no serviço de urgência e emergência de um hospital quartenário e filantrópico da região de Curitiba. R. Saúde Públ. Paraná. 2019 Jul.;2(Suppl 1): 38-45

Co CoPYRIGHT Esta obra é disponibilizada nos termos da Licença Creative Commons - 4. 0
Internacional. É permitida a reprodução parcial ou total desta obra, desde que citada a fonte. 
RESUMO O presente estudo relata a implantação de um protocolo de classificação de riscos no Pronto Atendimento de um hospital quaternário e filantrópico da região de Curitiba, baseado no modelo sul-africano. Esse modelo traz, além da sintomatologia relatada pelos usuários, a aferição dos sinais vitais para o auxílio da priorização do atendimento pelo profissional Enfermeiro, eliminando a subjetividade da sua prática e inserindo critérios baseados em evidências científicas e no perfil da Instituição. Após a implantação do Modelo Sul-Africano de classificação de risco no serviço de pronto atendimento foi observada melhorias significativas no atendimento.

PALAVRAS-CHAVE: Triagem. Gestão de risco. Enfermagem. Atendimento de Emergência

\begin{abstract}
The present study reports the implementation of a risk classification protocol in the Emergency Care of a quaternary and philanthropic hospital in the region of the city of Curitiba, based on the South African model. This model brings, along with the symptoms reported by the users, the measurement of the vital signs to help in the prioritization of care by the nurse, eliminating the subjectivity of its practice and inserting criteria based on scientific evidence and on the profile of the institution. After the implementation of the South African Model of risk classification in the emergency care service, significant improvements in care were observed.
\end{abstract}

KEYWORDS: Screening. Risk Management. Nursing. Ambulatory Care.

\title{
INTRODUÇÃO
}

$\mathbf{N}$

o Brasil, a realidade de uma grande parcela dos serviços de pronto atendimento e pronto-socorro chama a atenção pela falta de qualificação dos profissionais que prestam o cuidado, os quais estão na ponta da assistência; subjetividade na avaliação dos sinais e sintomas dos pacientes e; superlotação - muito pela busca direta dos usuários, os quais não possuem acesso imediato a serviços especializados e a tecnologias diagnósticas. A soma desses fatores contribui para o aumento do tempo de espera no atendimento, insatisfação da equipe multiprofissional com a função e, principalmente, contribui com o aumento da morbimortalidade dos usuários em estado crítico, uma vez que necessitam esperar por atendimento junto aos demais $^{1-3}$.

Para a realização do "pronto" atendimento aos casos de urgência e emergência faz-se necessária uma triagem minuciosa, baseada em elementos palpáveis e documentáveis. A triagem é um sistema de gerenciamento de risco clínico implantado em todo o mundo para gerenciar fluxo de paciente com segurança ${ }^{3}$.

O Plano de Humanização proposto pelo 
Ministério da Saúde em 2004, após a lei de implantação de sistemas de classificação de riscos em prontos atendimentos e prontossocorros, datada de 2002, trouxe alguns pilares para o atendimento nos serviços de emergência. Essa ação teve o intuito de alterar a realidade com vistas à resolutividade e humanização do atendimento com acolhimento individualizado, além de fortalecer a relação usuário/ trabalhador ${ }^{1,4}$.

Tal estratégia reflete diretamente no atendimento em tempo hábil para contenção de danos aos usuários de acordo com sinais e sintomas de base, melhorando a intervenção frente a situações de urgência e emergência que necessitam de uma ação iminente, não mais por ordem de chegada ou critérios de idade, por exemplo ${ }^{4}$.

Além disso, um sistema de classificação eficaz auxilia o profissional de saúde na realização de uma avaliação mais objetiva baseada em evidência científica, para a priorização ou não de um usuário frente a outro, com maior segurança e assertividade.

Outra ação importante, oriunda a essa implementação, é a educação da comunidade com relação à finalidade dos prontos atendimentos e prontos-socorros por ressaltar a prioridade da consulta frente à criticidade dos usuários.

No mundo inteiro existem vários modelos voltados à triagem dos usuários de serviços de urgência e emergência, como o Sistema de Triagem de Manchester (1997), a Escala Canadense de Triagem (1999), Escala Australiana de Triagem (2000), a Escala Americana Emergency Severity Index $(2000)^{5}$ e, mais recentemente, a Triagem Sul-Africana (2004) ${ }^{3}$.

No Brasil, o Estado de Minas Gerais implantou em seus serviços, em 2008, o
Protocolo de Manchester 2008 e, frente à necessidade de uma organização das triagens, demais estabelecimentos assistenciais de saúde aderiram à mesma metodologia. O diferencial dessa classificação, comparada às demais Escalas, é a não utilização de diagnóstico para a sua avaliação e, sim, a queixa apresentada, pois define o atendimento ao usuário pela sua criticidade momentânea e estabelece uma ferramenta sensivel para detectar situações críticas em usuários que necessitam de cuidados imediatos. O Protocolo de Manchester inclui classificação dos pacientes por cores relacionadas à criticidade do atendimento pela queixa principal, o que define o tempo de espera pela consulta médica. $\mathrm{O}$ protocolo é dividido em cinco níveis: vermelho/emergência, laranja/muito urgente, amarelo/urgente, verde/pouco urgente e azul/não urgente ${ }^{3,4}$.

Entretanto é um sistema patenteado e de alto custo para a maioria das instituições de saúde e possui falhas com relação ao acompanhamento dos usuários já triados, ou seja, não observa os casos com deterioração dos sinais vitais, uma vez que não prevê a reavaliação destes por parte da enfermagem ${ }^{4}$.

\section{MODELO SUL-AFRICANO}

Ao analisar outros protocolos de classificação de risco em pronto atendimento, observa-se que o modelo sul-africano é o mais aplicável e adaptável à realidade brasileira, uma vez que não se baseia apenas nos sintomas e queixas principais dos usuários, mas também nos sinais vitais, o que permite melhor assertividade na detecção das prioridades de atendimento. Assim, o protocolo baseia-se em duas avaliações, realizadas por profissional enfermeiro. No primeiro instante o enfermeiro coleta os dados 
quanto às queixas, sinais e sintomas, com ênfase no que fez o paciente procurar o atendimento, e na sequência é realizado o registro dos sinais vitais, que, se alterados, terão uma importância no desfecho da criticidade do usuário e, assim, na classificação do seu atendimento (Quadro 1 e 2) ). $^{3}$

Quadro 1 Escore de pontuação do Modelo Sul-Africano.

\begin{tabular}{|l|l|l|l|l|l|l|l|}
\hline \multicolumn{7}{|c|}{ Escore de Pontuação } & ( \\
\hline Pontos & $\mathbf{3}$ & $\mathbf{2}$ & $\mathbf{1}$ & $\mathbf{0}$ & $\mathbf{1}$ & $\mathbf{2}$ & $\mathbf{3}$ \\
\hline Mobilidade & & & & Deambula & com ajuda & Imóvel & \\
\hline Respiração & & $<9$ & & 9 a 14 & 15 a 20 & 21 a 29 & $>29$ \\
\hline \hline Frequência Cardíaca & & $<41$ & 41 a 50 & 51 a 100 & 101 a 100 & 111 a 129 & $>129$ \\
\hline Pressão sistólica & $<71$ & 71 a 80 & 81 a 100 & 101 a 199 & & $>199$ & \\
\hline Temperatura & & & & 36 a 38 & & $>38,5 \%$ & \\
\hline Consciência & & & & Alerta & Rege a voz & Reage a dor & Não responde \\
\hline Oxigenação & & & & $>94 \%$ & $<94 \%$ & & \\
\hline Trauma & & & & não & sim & & \\
\hline
\end{tabular}

Fonte: Cristino (2014)

Quadro 2 Discriminadores e escore de pontuação

\begin{tabular}{|c|c|c|c|c|c|}
\hline Nível-cor & Azul (1) & Verde (2) & Amarelo (3) & Laranja (4) & Vermelho (5) \\
\hline Tempo máximo & 240min & 120min & $30 \mathrm{~min}$ & 10min & imediato \\
\hline Escore & 0 & 1 a 2 & 3 a 4 & 5 a 6 & $>7$ \\
\hline Dor & 0 a 1 & 2 a 4 & 5 a 7 & 5 a 10 & \\
\hline Trauma & & & & Queda, acidente & \\
\hline Consciência & & & & Reduzida & Inconsciente \\
\hline Convulsão & & & Chega acordado & Sonolência & Inconsciente \\
\hline Sangramento & & & Pequena monta & Grande monta & Choque \\
\hline Déficit motor & & & & Déficit >6h & Déficit $<6 h$ \\
\hline Queimadura & & & & & Face, inalação \\
\hline Intoxicação & & & & Acordado & Inconsciente \\
\hline Imunológico & & & & Ciancer & \\
\hline Diabete & & & & Glicemia >400 & Glicemia <70 \\
\hline Dor torácica & & & & Todos nível 4 & \\
\hline
\end{tabular}

Fonte: Cristino (2014) 
Após a análise dos itens é verificado o posicionamento das queixas e a faixa dos sinais vitais dentro de escores, os mais altos são os usuários mais críticos, os menores, menos críticos. A classificação é realizada em cinco níveis, assim como o Protocolo de Manchester. Uma diferenciação bastante relevante frente a esse protocolo é a necessidade de realizar a reavaliação dos usuários, a qual ocorre sempre após extrapolar os tempos estabelecidos, quando houver necessidade de nova aferição dos sinais vitais como temperatura, ou qualquer alteração clínica importante evidenciada pela equipe (vômitos, desmaios entre outros). Assim, a criticidade é baseada em informações que permitem classificar o paciente em uma das cinco categorias e estabelece o tempo máximo para a avaliação médica. Por conseguinte, o acolhimento deixa de ser subjetivo e passa a ser baseado em critérios bem estabelecidos3.

Dessa forma, a instituição objetivou:

1. Implantar um sistema de classificação de risco no Pronto Atendimento adequado à realidade do hospital;

2. Promover maior segurança aos Enfermeiros do setor de Triagem a fim de proporcionar uma metodologia mais concreta para a priorização dos atendimentos de urgência e emergência.

O presente artigo tem por objetivo relatar a experiência da implantação do Sistema de Classificação de Risco Sul-Africano em um hospital filantrópico, de grande porte, situado na cidade de Curitiba.

\section{CENÁRIO E IMPLANTAÇÃO}

A implantação do Sistema de Classificação de Risco Sul-Africano ocorreu em um Hospital Geral de grande porte, quaternário, localizado na cidade de Curitiba, considerado um dos maiores centros de saúde do Estado do Paraná. Tratase de um hospital filantrópico, mantenedor de um grupo hospitalar responsável por mais de 70\% de atendimentos do Sistema Único de Saúde (SUS) em suas unidades. Fundado em 1953, inicialmente, o hospital possuía como principal objetivo disseminar o conhecimento e cuidar dos mais necessitados, baseado no carisma vicentino. Em 2002, por problemas financeiros e com grande risco de fechar suas portas, a estratégia de gestão do hospital foi alterada, passando a atender apenas usuários particulares e conveniados. Com a mudança no perfil de atendimento, o hospital passa a adquirir os recursos necessários para manter os demais hospitais administrados pelas Irmãs Vicentinas. Hoje, o hospital possui 243 leitos, desses 50 leitos são de Unidade de Terapia Intensiva, sendo 30 adultos e 20 neopediátricos. É um hospital referência no Brasil e no mundo pela qualidade em tratamentos clínicos e cirúrgicos de alta complexidade, como Transplante de Medula Óssea e Hepático, além de ser referência no atendimento oncológico e em gestantes de alto risco.

Por prezar pela qualidade e segurança no atendimento, desde 2001 conta com programas e serviços internos os quais disseminam conceitos de melhorias de processos e assistência. É acreditado pela Organização Nacional de Acreditação (ONA) desde 2003, com nível máximo de reconhecimento por essa Instituição.

Na busca por melhorias, sua gestão busca constantemente rever seus processos com a 
avaliação de suas estratégias. Assim, observouse que o Pronto Atendimento necessitava de processos mais seguros, não apenas aos usuários como também aos profissionais de enfermagem. Mediante o resultado de suas avaliações, a gestão buscou melhorar e aprofundar o conhecimento dos profissionais do setor; mudar o perfil dos funcionários, preencher as vagas em aberto com trabalhadores com maior experiência na área de enfermagem; além de rever as ferramentas utilizadas para a classificação dos usuários. Tais alterações foram consideradas como importantes e necessárias, pois o serviço de urgência e emergência da instituição é um dos maiores de Curitiba, sendo o segundo em atendimento pediátrico. Atualmente são atendidos, em média, oito mil usuários/mês, sendo 1.500 adultos e gestantes e 6.500 crianças.

Foram analisadas algumas metodologias para a implantação de ferramentas que potencializassem a triagem e, consequentemente, trouxesse maior assertividade no procedimento e identificação das urgências e emergências. Todavia, observou-se que nenhuma se adequava às especificidades da pediatria e muito menos da instituição. Com isso, os modelos mais conhecidos, como Manchester, foram descartados. Com auxílio de uma consultoria externa contratada, chegou-se então ao modelo de triagem SulAfricana

\section{IMPLANTAÇÃO}

Desde abril de 2018 foi estabelecido um grupo de condução e estudo sobre a implantação de um modelo de classificação de riscos para o serviço de urgência e emergência da instituição que utilizasse itens mais objetivos para assegurar aos profissionais de saúde segurança em sua avaliação e, com isso, auxiliar na redução de mortalidade e reduzir o tempo de espera dos usuários. O grupo teve como participantes o Gerente Médico do Hospital, a Coordenadora de Enfermagem do Pronto Atendimento, Coordenadora Médica do Pronto Atendimento e uma Analista de Risco. Reuniões semanais aconteceram conforme o cronograma estabelecido e o plano de ação traçado.

Em junho do mesmo ano, após analisar todos os modelos de triagem e, por incentivo da própria empresa de consultoria do hospital, optou-se pela implantação do modelo de triagem Sul-Africano. Além de ser um modelo que permite melhor avaliação, ele não tem custos e é flexível na descrição dos sintomas e queixas principais, o que possibilita adaptação ao perfil epidemiológico da instituição. Após a escolha, foi solicitada ao serviço de informática uma avaliação para verificar a possibilidade de inserir e parametrizar os descritores, os sinais vitais e, consequentemente, o cálculo dos escores no sistema interno, com o objetivo de automatizar o processo. 0 intuito dessa ação foi desenvolver uma barreira ao profissional de saúde para evitar qualquer interpretação dos dados de forma subjetiva.

Esta etapa estendeu-se até final de agosto de 2018 e, durante esse período, toda a equipe de enfermagem, recepção e segurança foi submetida a capacitações para a aplicação do protocolo e acolhimento do usuário. Foi divulgado material informativo junto à comunidade, como cartazes e quadros sobre o tema, e foi concluído o próprio protocolo descritivo.

Em setembro, deu-se início ao processo de classificação na triagem dos usuários do hospital. Observou-se junto aos médicos uma redução no tempo de espera da triagem. Contudo, os médicos questionaram tal processo, principalmente o serviço infantil, pois aumentou 
o número de pacientes triados como verde, ou seja, de baixa urgência, frente aos de nenhuma urgência (azul), o que implicou diretamente no aumento do tempo de espera para a consulta médica.

Em reunião do grupo observou-se que os médicos não haviam sido treinados sobre o protocolo, e, por isso, houve questionamentos sobre a avaliação da criticidade. Outros problemas identificados foram com relação aos sinais vitais da parte pediátrica como, por exemplo, a febre. O paciente infantil que chega com febre possui alteração de sinais vitais e isso acarreta em uma classificação mais crítica, decorrente apenas do quadro febril. Assim, foi revisado o protocolo de hipertermia e reparametrizado o sistema de classificação para coloração azul e nova triagem, após 30 minutos, ou de acordo com o tempo de efeito da medicação ministrada. Alguns parâmetros para a classificação de risco em gestantes também estão sendo revisados.

Em outubro/2018, após um mês da implantação da classificação de risco na triagem, observaram-se resultados relevantes como o cumprimento dos acordos de tempo para os protocolos gerenciáveis, como Acidente Vascular Cerebral (Porta tomografia dentro do tempo estipulado de 45 minutos) e Dor Torácica (Porta eletrocardiograma dentro do tempo estipulado de 10 minutos); diminuição em 25\% do tempo de recepção/triagem, de 12 minutos para 9 minutos, abaixo da média estimada pela literatura, que é de 10 minutos $^{6}$; e redução do tempo médio de espera para o atendimento em consultório. Esse último sofreu redução de 42\%, passando de 38 minutos para em média de 22 minutos no pronto atendimento adulto.

\section{CONSIDERAÇÕES FINAIS}

Possuir um sistema de classificação empodera os profissionais de enfermagem no desempenho de seu papel, uma vez que qualifica sua avaliação e, consequentemente, melhora a atuação no desfecho clínico do usuário. Ainda, contribui com a redução da mortalidade e permite aumentar o grau de satisfação junto ao serviço e à comunidade.

Algumas alterações e ajustes ainda são necessários, como definir melhor os critérios para a reclassificação dos pacientes. Porém, ficou evidente que os sinais vitais continuam sendo a melhor ferramenta para avaliação do quadro de deterioração clínica dos usuários. Por isso, de nada adianta os estabelecimentos assistências de saúde possuírem ferramentas ou métodos de análises eficazes, se a aferição de dor e/ou frequência respiratória, cardíaca, pressão arterial e temperatura continuarem a ser negligenciadas. A instituição defende como verdadeira, mediante a implantação do Modelo de Classificação Sul Africano, a premissa "menos é mais", principalmente no âmbito da qualidade do cuidado. 


\section{REFERÊNCIAS}

1. Prudêncio CPG, Monteiro RAN, Ribeiro BCM, Gomes MSM, Manhães LSP. Percepção de enfermeira(o)s sobre acolhimento com classe de risco no serviço de pronto atendimento. Revista Baiana de Enfermagem [internet]. 2016 [citado 2018 out 15]; 30(2):1-10. Disponivel em: https://portalseer.ufba.br/index.php/enfermagem/ article/view/14917/pdf_46

2. Souza SS. Acolhimento e classificação de risco: proposta de material educativo para um hospital de ensino. Florianópolis. Monografia [Especialização em Linhas de Cuidado em Enfermagem - Urgência e Emergência] - Universidade Federal de Santa Catarina:2014

3. Cristino TGT. Avaliação e Classificação de risco baseada no sistema de triagem Sul-Africano adaptada em um modelo de serviço de emergência: um estudo descritivo e observacional em um hospital terciário privado. In: Congresso Nacional de excelencia em gestão; 2014 ago 08-09; Rio de Janeiro, Brasil;2014.

4. Souza CC, Toledo AD, Tadeu LFR, Chianca TCM. Classificação de risco em pronto-socorro: concordância entre um protocolo institucional brasileiro e Manchester. Rev. Latino-Am. Enfermagem [internet]. 2011 [citado 2018 out 15]: 19(1):[8 telas]. Disponivel em: http://www.scielo.br/pdf/rlae/v19n1/pt_05.pdf

5. Costa MAR, Versa GLGS, Bellucci JA, Inoue KC, Sales AC, Matsuda LM. Acolhimento com classificação de risco: avaliação de serviços Hospitalares de Emergência. Esc Anna Nery [nternet]. 2015 [citado 2018 set 18]: 19(3):491-497. Disponivel em: http:// www.scielo.br/pdf/ean/v19n3/1414-8145-ean-19-03-0491.pdf.

6. Magalhães-Barbosa MC, Prata-Barbosa A, Cunha AJLA, Lopes CS. CLARIPED: um novo instrumento para classificação de risco em emergências pediátricas. Rev. Paul Pediatr. [internet] 2016: 34(3):254-262. Disponivel em: http://www.scielo.br/pdf/rpp/ v34n3/pt_0103-0582-rpp-34-03-0254.pdf 